RESEARCH ARTICLE

\title{
Presumptive Diagnosis of Buruli Ulcer Based on Clinical Presentations
}

\author{
Paul Eniola Oluniyi ${ }^{1}$, Adewale Adegboyega Oke ${ }^{1}$, Isaac Omotosho Komolafe ${ }^{1}$
}

\section{ABSTRACT}

Objectives: Buruli ulcer disease (BUD) is a neglected tropical disease of the skin, subcutaneous tissue and occasionally bone. Except in endemic areas, the diagnosis of BUD can be a challenge. This study was an attempt to presumptively identify cases of Buruli ulcer in the absence of a laboratory confirmation.

Methods: A presumptive diagnosis of Buruli ulcer by directly comparing clinical presentations with WHO-confirmed pictorial images of the disease was carried out on patients with suspected Buruli ulcers presenting to two government-owned hospitals in Ogun State, South-West, Nigeria.

Results: Eighteen suspected cases of BUD from as many outpatients were identified within a three-month period, comprising $13(72.2 \%)$ females and five (27.8\%) males. $10(55.6 \%)$ and eight $(44.4 \%)$ of them were from the tertiary and secondary health facilities, respectively. Eleven (61\%) of the ulcers were located on the leg, four $(22 \%)$ on the breast and four (22\%) on the hand. $14(77.8 \%)$ of the patients were above 40 years of age, two $(11.1 \%)$ were between 18 and 30 years while only two (11.1\%) were below 15 years. $16(89 \%)$ of the patients could not remember how the ulcer started while two (11\%) of them said their lesions started with a scratch. $7(39 \%)$ of the ulcers were painless; $11(61 \%)$ were minimally painful while $15(83 \%)$ had undermined edges which are presentations consistent with Buruli ulcer disease.

Conclusions: The detection of these ulcers in just two hospitals and within a period of three months is significant and suggestive of Buruli ulcer being probably more prevalent in South-west Nigeria than aforethought. $J$ Microbiol Infect Dis 2017; 7(1): 36-41

Keywords: Buruli ulcer, presumptive diagnosis, clinical presentations, Ogun State, Nigeria

\section{INTRODUCTION}

Buruli ulcer disease (BUD) which is caused by Mycobacterium ulcerans is an indolent, necrotizing infection of the skin, cutaneous tissue and occasionally involving bones [1]. This neglected disease regarded as the third most mycobacterial disease of humans after tuberculosis and leprosy, primarily affects the poor in remote and rural areas particularly those living in wetlands, around stagnant lakes or slow-flowing streams [2].

Buruli ulcer which has been reported in over 30 countries in tropical and subtropical regions of the world has become an emerging infectious disease of major concern in West and Central Africa in countries like Ghana, Benin Republic, Cote d'Ivoire, Togo, Democratic Republic of Congo (DRC), Congo-Brazzaville and
Cameroon [3]. In some parts of Cote d'Ivoire, Ghana and Benin Republic, Buruli ulcer incidence has overtaken tuberculosis and leprosy as the most common [4]. However, the picture of Buruli ulcer in Nigeria is less clear in spite of the fact that the first cases of BUD in the country were reported in 1967 by Gray et al [5].

Then in 1975, Oluwasanmi et al [6] confirmed a case of the disease among a Caucasian family who lived close to a newly constructed dam on the campus of the University of Ibadan in SouthWest Nigeria. This led to the identification of 23 more cases within and around Ibadan metropolis. Thereafter there was no follow-up search or research on Buruli ulcer in Nigeria for over two decades.

Between 1998 and 2000, the Institute of Tropical Medicine in Belgium confirmed Buruli ulcer

\footnotetext{
${ }^{1}$ Department of Biological Sciences, Microbiology Unit, College of Natural Sciences, Redeemer's University, Ede, Osun State, Nigeria
}

Correspondence: Adewale Adegboyega Oke, Microbiology Unit, Department of Biological Sciences, Redeemer's University, Off Gbongan-Oshogbo Road, Ede, Osun State, Nigeria, Email address: okead@run.edu.ng Received: 13 March 2016, Accepted: 26 December 2016

Copyright (C) JMID / Journal of Microbiology and Infectious Diseases 2017, All rights reserved 
cases from samples sent to it from the Leprosy and Tuberculosis Hospital in Moniaya-Ogoja, Cross River State, Nigeria [7].

In 2006, the World Health Organization (WHO) experts in conjunction with the national and state health authorities in Nigeria embarked on a 5day BUD case search in Akwa lbom, Cross Rivers, Anambra, Ebonyi and Enugu States (South-South and South-East regions in Nigeria). Out of the 37 suspected specimens harvested from these areas and examined at the Institute of Tropical Medicine, Antwerp, Belgium using the IS2404 PCR method, 14 (38\%) was confirmed to be Buruli ulcer [8].

This result of the WHO technical team to Nigeria in 2006 was an eye opener coupled with the fact that patients from Nigeria mostly those living near the border with Benin Republic and Cameroon have been reportedly treated for Buruli ulcer in those countries [8-10]. A recent retrospective study of data of PCR- confirmed Nigerian patients with Buruli ulcer treated in Benin Republic gives credence to this fact [11]. The endemicity of the disease in the neighboring countries also makes it highly probable that $\mathrm{BU}$ could be endemic, in some yet to be identified areas in Nigeria.

In Nigeria, information about Buruli ulcer is scanty not only among the populace but also among healthcare givers with the consequent effect on their inability to correctly identify Buruli ulcer cases resulting in low surveillance, low monitoring, low reporting, poor infection prevention and control coupled with inadequate public health infrastructure. This study presents the results of a presumptive diagnosis of Buruli ulcer based on the clinical presentations of suspected cases in two public hospitals in SW Nigeria.

An earlier questionnaire-based study carried out by us to assess the level of awareness of Buruli ulcer among the relevant medical professionals in Lagos, Ogun and Oyo States in SW Nigeria was an eye-opener. Although, Buruli ulcer is a neglected tropical disease, it is quite shocking and interesting to discover that most medical personnel who are supposed to be caregivers know little or nothing about the disease and cannot recognise the disease even if presented with a typical case. The present study, apart from creating BUD awareness among health givers, is an attempt to presumptively diagnose Buruli ulcer, based solely on clinical presentation, as an aid to the laboratory confirmation of the disease.

\section{METHODS}

\section{Ethical Clearance}

The Ethical Review Committee of the State Hospital, ljebu-Ode and the Teaching Hospital Sagamu, Ogun State approved the study. Written informed consent was obtained from all participants in the study and participation was voluntary. Parents of under-aged participants gave written informed consents on behalf of their wards. Collection of personal and pertinent information from the patients was done in both hospitals under the supervision of staff members. Patients' names were replaced with numbers to ensure confidentiality.

\section{Study place}

This study was carried out within three months at the Olabisi Onabanjo University Teaching Hospital (OOUTH), Sagamu and the State Hospital, ljebu-Ode, both in Ogun State, SouthWest Nigeria. Both are state government-owned hospitals that serve the general public. While OOUTH is a tertiary healthcare facility the State Hospital is a secondary level healthcare facility. Both Sagamu and ljebu-Ode are urban towns located some $60 \mathrm{~km}$ and $91.8 \mathrm{~km}$ from Lagos while they are both $133 \mathrm{~km}$ and $165 \mathrm{~km}$ from the Benin Republic border respectively (S1 Fig.).

\section{Study design and methodology}

A color atlas of Buruli ulcer images from WHO documents was prepared (S2 Fig.) and mounted at the out-patients department (OPD) of the hospitals, to serve as guide in the identification of similar lesions presenting to the hospitals. Patients presented with various wounds to the OPD of these hospitals were approached and interviewed. Patients with ulcers that originated from automobile and domestic accidents including burns were excluded from the study while those with ulcers of uncertain causation were enrolled to participate in the study. Such ulcers that presented to the hospitals were directly compared with the images of the atlas and visual observations were made as appropriate. Photographs of suspected cases 
were taken after obtaining consent from the participants.

Personal and pertinent information which included, sex, age, residence, duration of ulcer, location of ulcer, probable origin of ulcer and whether or not the patients felt pain, were collected from the participants.

\section{RESULTS}

Within the period of this study a total number of 147 patients presented with various wounds to the hospitals. $129(88 \%)$ were associated with automobile and domestic accidents while 18 (12\%) patients with BUD-like ulcers (all at the ulcerative stage) were observed. Ten (10/56\%) were from OOUTH in Sagamu and eight (8/44\%) from the State Hospital, ljebu-Ode. Of the 18 patients, $13(72.2 \%)$ were females and 5 $(27.8 \%)$ were males; $11(61 \%)$ of the ulcers were located on the leg, 4 (22\%) on the breast and $4(22 \%)$ on the hand. $14(79 \%)$ of the patients were adults above 40 years of age, 2 (11\%) were between ages 18 and 30 years and only $2(11 \%)$ were children below 15 years of age. $11(61 \%)$ said their ulcers were minimally painful while 7 (39\%) experienced no pain at all. $15(83 \%)$ ulcers had observable undermined edges although only 4 of the ulcers were shown (Figure 1). As for duration of the ulcers, 4 $(22.2 \%)$ were above 1 year including one of 20 years duration while $14(77.8 \%)$ were below one year (Tables I and 2).

Table 1. Information about patients enrolled at OOUTH, Sagamu.

\begin{tabular}{lcccccc}
\hline S/N & Residence & Sex/Age & Duration of ulcer & Location of ulcer & Probable source of ulcer & Pain \\
\hline 1 & Sagamu & F/22 & $<1$ year & Breast & DK & \pm \\
$\mathbf{2}$ & Sagamu & F/12 & $<1$ year & Leg & Scratch & \pm \\
$\mathbf{3}$ & Sagamu & F/45 & Long period & Leg & CR & \pm \\
$\mathbf{4}$ & Sagamu & M/47 & $<1$ year & Leg & DR & \pm \\
$\mathbf{5}$ & Sagamu & F (Adult) & Uncertain & Breast & Scratch & - \\
$\mathbf{6}$ & Sagamu & M/76 & 2 years & Heel & DK & - \\
7 & Sagamu & F/64 & $<1$ year & Breast & CR & \pm \\
$\mathbf{8}$ & Sagamu & F/Adult & $<1$ year & Leg & DK & \pm \\
$\mathbf{9}$ & Sagamu & F/45 & $\approx 20$ years & Leg & CR & \pm \\
$\mathbf{1 0}$ & Sagamu & M/43 & $<1$ year & Leg & DK & - \\
\hline
\end{tabular}

Table 2. Information about patients enrolled at the State Hospital, ljebu-Ode.

\begin{tabular}{ccccccc}
\hline S/N & Residence & Sex, Age $(\mathrm{y})$ & Duration of ulcer & Location of ulcer & Probable Source of ulcer & Pain \\
\hline 1 & ljebu-Ode & F, 60 & $<1$ year & Leg & DK & \pm \\
2 & ljebu-Ode & F, 75 & $<1$ year & Leg & DK & - \\
3 & ljebu-Ode & F, 56 & $<1$ year & Breast & CR & \pm \\
4 & ljebu-Ode & F, 62 & $<1$ year & Hand & DK & \pm \\
5 & ljebu-Ode & M, 84 & $<1$ year & Hand \& Leg & DK & - \\
6 & ljebu-Ode & M, 25 & $<1$ year & Hand & DK & - \\
7 & ljebu-Ode & F, 66 & $<1$ year & Leg & DK & \pm \\
8 & ljebu-Ode & F, 1 & $?$ & Hand & $?$ & - \\
\hline
\end{tabular}



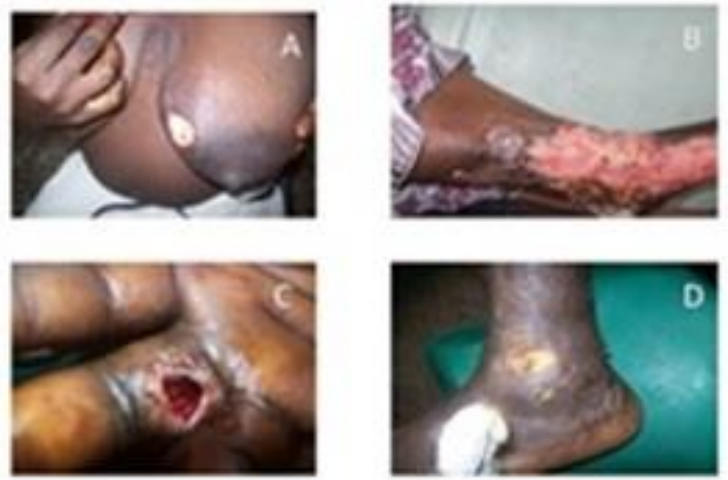

Figure 1. Some of the ulcers that presented to the two hospitals in Ogun State.

A. Buruli ulcer-like ulcer on the breast of a female patient at OOUTH, Sagamu.

B. Buruli ulcer-like ulcer on the leg of a male patient at OOUTH, Sagamu

C. Buruli ulcer-like ulcer on the hand of a male patient at State Hospital, ljebu-Ode.

D. Buruli ulcer-like ulcer on the leg of a male patient at State Hospital, ljebu-Ode.
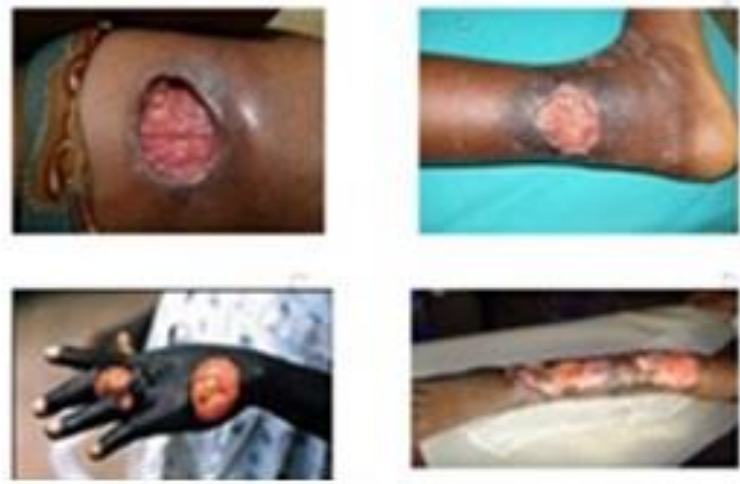

Figure 2. Album of some WHO-confirmed BUD images.

\section{DISCUSSION}

Although Buruli ulcer was first described in Nigeria in 1967 [5] and several other documented reports were made in 1976 [6], 2007[8] and 2015 [11], the disease still remains largely unknown in most parts of the country till date. In the available epidemiological data in the West African sub-region which has now become the epicentre of BUD, Nigeria presents a notable gap in cases, compared with Benin Republic and Cameron which have highly endemic regions $[10,11]$. This gap may be due to lack of awareness of the disease among both the general public and healthcare givers leading to the misdiagnosis of disease, under-reporting and treatment failure [8].
Except in endemic areas, the diagnosis of Buruli ulcer can be a challenge. However, in this study we directly compared the WHO-confirmed BUD images with cases being presented to two hospitals in Ogun State as an aid to the presumptive diagnosis of the disease in an apparently non-endemic BUD area of Ogun State [11].

A total of 18 patients with BU-like ulcers presented to the hospitals during the study period of which $13(72.2 \%)$ were females and only $5(27.8 \%)$ were males. This is at variance with the available statistics elsewhere in Africa where males predominate [12] even though in correlation with a similar study in Japan [13]. Fifteen $(83 \%)$ of the lesions were on the limbs with $11(61 \%)$ occurring on the lower limbs which is in conformity with previous studies [2]. However, unlike in other studies in Africa where children under 15 years of age predominate $[10,11]$ only $2(11 \%)$ of the patients in our study were children which correlates with some studies in Australia and Japan where BUD patients under 15years of age accounted for only $10 \%$ and $19 \%$ respectively [14]. However, the results of this study could probably have been different if the study period had been extended to allow more BUD-like cases present to the hospitals. Like in Buruli ulcer cases 16 $(89 \%)$ of the patients in this study did not know or could not remember how the ulcers started while only $2(11 \%)$ attributed the origin of their ulcers to a scratch.

Virtually all the ulcers observed in this study were already in the ulcerative stage which is similar to available statistics provided in other parts of the world. For example, in Australia and Japan, less than $10 \%$ of cases are diagnosed before ulceration [15]. Furthermore, lack of knowledge of BUD and thus wrong diagnosis on the part of healthcare personnel make them treat all ulcers alike. Nurses in the dressing room for example, make use of materials like normal saline, honey, purit, uzol lotion, hydrogen peroxide and savlon [16] in treating wounds. These might be successful in treating other wounds but not Buruli ulcer which can only respond to antibiotic combination therapy administered over a period of eight weeks $[14,15]$ and this inappropriate treatment could probably be the reason for the long duration of the ulcers which are still not healing (Tables 1 
and 2). It was also observed in this study that $7(39 \%)$ of the ulcers were painless while the rest were minimally painful which is another characteristic feature of Buruli ulcer [17].

However, the fact that these ulcers were detected in areas not primarily associated with BUD in Ogun State is significant, meaning that this disease might have a much wider spread than afore-thought.

Presumptive diagnosis of BUD based on clinical presentation as an aid to disease diagnosis is good but not enough as every suspected case must be confirmed by laboratory protocols particularly by deploying the PCR technique. This we could not carry out in this study for logistic reasons. The value of this study however, is in creating awareness that BUD might be more prevalent in Ogun State than earlier thought, thus corroborating the reports of Marion et al[11] that a cohort of 127 patients from towns and villages in Ogun State that border Benin Republic had crossed into that country and were treated for BUD over a period of ten years. The fact that 18 ulcers that simulate classical Buruli ulcer images as documented by the WHO were observed within a period of 3 months in this study provides a solid basis for an enlarged Buruli ulcer case search to cover not only Ogun State but all the six states in SW Nigeria, bearing in mind that 24 BUD cases had earlier been reported from Ibadan in Oyo State [6] which is contiguous with Ogun State.

Acknowledgements: We want to sincerely thank the Management and staff of Olabisi Onabanjo University Teaching Hospital (OOUTH), Sagamu and the State Hospital, ljebu-Ode for giving us the permission to carry out this project in their hospitals, particularly Mrs V.T. Babalola and Mrs. O. Adepitan for their technical assistance.

Declaration of conflicting interests: The authors declared no conflicts of interest with respect to the authorship and/or authorship of this article.

\section{REFERENCES}

1. Bratschi MW, Ruf M, Andreoli $A$, et al. Mycobacterium ulcerans Persistence at Village water Source of Buruli Ulcer Patients. PLoS Negl Trop Dis 2014; 8(3):e2756.

2. Komolafe OO. Buruli ulcer in Malawi - a first report. Malawi Med J 2001; 13(3): 37-39.

3. Walsh DS, Portaels F, Meyers WM. Buruli ulcer: Advances in understanding Mycobacterium ulcerans infection. Dermatol Clin.2011; 29(1): 1-8.

4. Asiedu K, Raviglione M, Scherpbier R. Buruli ulcer (Mycobacterium ulcerans infection) (WHO/COS/CPE/GBUI/2000.1) Geneva: WHO (2000).

5. Gray HH, Kingma S. Mycobacterial skin ulcers in Nigeria. Trans R Soc Trop Dis Hyg1967; 61(5):71214.

6. Oluwasanmi JO, Solankee TF, Oluri EO, Itayemi SO, Alabi GO, Lucas AO. Mycobacterium ulcerans (Buruli) skin ulceration in Nigeria. Am J Trop Med Hyg 1976; 25:122-128.

7. Janssens PG, Pattyn SR, Meyers WM, Portaels F. Buruli ulcer: an historical overview with updating to 2005. Bulletin des séances Académie Royale des Sciences d'outre-mer, Brussels 2005; 51:165-159.

8. Chukwuekezie O, Ampadu E, Sopoh G, et al. Buruli ulcer, Nigeria (Letter). Emerg Infect Dis 2007; 13 (5): 782-783.

9. Debackers M, Aguiar J, Steunou C. Mycobacterium ulcerans disease (Buruli ulcer) in rural hospital, Southern Benin (1997 - 2001). Emerg Infect Dis 2004; 10:1391-1398.

10. Noeske J, Kuaban C, Rondini S. Buruli ulcer disease in Cameroon rediscovered. Am J of Trop Med Hyg 2004; 70: 520-6.

11. Marion E, Carolan K, Adeye A, Kempf M, Chauty A, Marsollier L. Buruli ulcer in South Western Nigeria: A Retrospective Cohort Study of Patients Treated in Benin. PLoS Negl Trop Dis 2015; 9(1): e3443.

12. Vincent QB, Ardant MF, Adeye A, et al. Clinical epidemiology of laboratory-confirmed Buruli ulcer in Benin: a cohort study. Lancet Glob Health 2014; 2(7): e422-430.

13. Nakanaga $K$, Hoshino $Y$, Yotsu RR, Makino M, Ishii N. Nineteen cases of Buruli ulcer diagnosed in Japan from 1980 to 2010. J Clin Microbiol 2011; 49(11):3829-3836

14. Nienhuis WA, Stienstra $Y$, Thompson WA, et al. Antimicrobial treatment for early, limited Mycobacterium ulcerans infection: a randomized controlled trial. Lancet 2010; 375(9715):664-672.

15. WHO. Treatment of Mycobacterium ulcerans Disease (Buruli ulcer): Guidance for health workers. Geneva 2012; 1-66. 
16. Velding K, Klis S, Abass KM, Tuah W, Stienstra Y, Werf T. Wound Care in Buruli Ulcer Disease in Ghana and Benin Am J Trop Med Hyg 2014; 91(2): 313-318.

17.de Zeeuw J, Alferink M, Barogui $Y T$, et al. Assessment and Treatment of Pain during Treatment of Buruli Ulcer. PLoS Negl Trop Dis.2015;9(9): e0004076. 